\title{
Producción de carbón vegetal a partir de arbustos nativos en la Región de Atacama, Chile
}

\section{Production of charcoal from native shrubs in the Atacama Region, Chile}

\author{
Rodrigo A. Estevez ${ }^{1,2}$, Francisco A. Squeo ${ }^{1,3,4}$, Gina Arancio ${ }^{1,3}$ \& Manuela B. Erazo ${ }^{5}$ \\ ${ }^{1}$ Instituto de Ecología y Biodiversidad (IEB), Casilla 653, Santiago, Chile. \\ ${ }^{2} \mathrm{PhD}$ Science Program, School of Botany, University of Melbourne, Parkville Victoria 3010 Australia. \\ ${ }^{3}$ Departamento de Biología, Universidad de La Serena, Casilla 599, La Serena, Chile. \\ ${ }^{4}$ Centro de Estudios Avanzados en Zonas Áridas (CEAZA), Casilla 599, La Serena Chile. \\ ${ }^{5}$ Escuela de Antropología, Universidad Academia de Humanismo Cristiano, Av. Condell 343, Santiago, Chile. \\ f_squeo@userena.cl
}

\begin{abstract}
RESUMEN
La producción de carbón vegetal a partir de arbustos leñosos es una de las principales amenazas para la vegetación nativa en zonas áridas. En este trabajo se estima la producción de carbón en la provincia de Huasco, al sur de la Región de Atacama, y se identifican las principales especies utilizadas en este proceso. Se detectaron 21 puntos de venta de carbón nativo en el área de estudio. En cada uno de ellos se recolectaron muestras del carbón comercializado, las que fueron posteriormente comparadas con muestras de referencia. Se realizaron 28 entrevistas a vendedores, productores de carbón nativo e informantes claves, para estimar el volumen de producción anual. Se identificaron 4 sitios productivos en la zona de estudio, tres de ellos entre Vallenar y El Tránsito, y uno en Incahuasi-Tres Cruces. Se estimó para el año 2007 una producción total de carbón vegetal de 281 toneladas para la provincia de Huasco, lo que equivale a una extracción anual de arbustos en un área de entre 573 y 1.112 hectáreas. Los principales arbustos nativos utilizados corresponden a Fabiana imbricata (Solanaceae), Balsamocarpon brevifolium (Fabaceae) y Adesmia hystrix (Fabaceae).
\end{abstract}

Palabras clave: Adesmia, Balsamocarpon, Fabiana, amenaza a la biodiversidad, zonas áridas.

\begin{abstract}
Charcoal production from woody shrubs is one of the main threats to native vegetation in arid zones. The aim of this investigation was to estimate the production of charcoal produced in Huasco province, southern of Atacama Region, and to identify the main species used in this process. Twenty one points of sale of native charcoal were detected in the study area. In each point, samples of the commercialized charcoal were collected and subsequently compared with reference samples. Twenty eight sellers, charcoal producers, and key informants were interviewed to estimate the volume of annual production. Four places of production were identified in the study area, three of them between Vallenar and El Tránsito, and one of them in Incahuasi-Tres Cruces. For 2007, a total production of 281 tons of charcoal was estimated in Huasco province, which is equivalent to an annual extraction of shrubs between 573 y 1,112 hectares. The main native shrubs used for the production of charcoal are Fabiana imbricata (Solanaceae), Balsamocarpon brevifolium (Fabaceae) and Adesmia hystrix (Fabaceae).
\end{abstract}

KeYwords: Adesmia, Balsamocarpon, Fabiana, threat to biodiversity, arid zones. 


\section{INTRODUCCIÓN}

En la zona árida del norte-centro de Chile (regiones de Atacama y Coquimbo) la vegetación nativa es dominada por cactáceas y arbustos deciduos de verano, y en menor medida, por arbustos siempreverdes (Fuentes 1988, Armesto et al. 1993). Los ecosistemas en zonas áridas y semiáridas se caracterizan por presentar un patrón de vegetación tipo mosaico, con parches de mayor biomasa y fertilidad, en los cuales los arbustos tienen un rol importante en su funcionamiento y estructura (Aguiar \& Sala 1999). A pesar de las variaciones climáticas inter-anuales e inter-decadales, estos ecosistemas presentan una gran estabilidad en el tiempo, como lo muestran estudios en la zona semiárida de Chile central y el Parque Nacional Bosque de Fray Jorge en la Región de Coquimbo (Fuentes et al. 1984, Gutiérrez et al. 1993). En paisajes con relieves accidentados propensos a la erosión por lluvia o viento, como en el norte-centro de Chile, la vegetación arbustiva contribuye a frenar el desgaste del relieve y la erosión causada por viento (Gutiérrez \& Squeo 2004). Los arbustos cumplen también un rol clave en el funcionamiento hídrico de estos ecosistemas. Especies con raíces pivotantes extraen agua desde las capas freáticas, transportándola a estratos superficiales y secos, en un fenómeno conocido como levantamiento hidráulico (Caldwell et al. 1998, Horton \& Hart 1998, León \& Squeo 2004, Squeo et al. 2006a). Estudios en el norte-centro de Chile muestran las implicancias positivas (facilitación) de este proceso en las interacciones planta-planta, sustentando la biodiversidad (Squeo et al. 1999, Squeo et al. 2009). En algunas situaciones, el follaje de la vegetación arbustiva también puede interceptar neblina costera proporcionando humedad en las capas superficiales del suelo (Gutiérrez \& Squeo 2004).

La extracción de arbustos en zonas áridas y semiáridas es una de las principales amenazas para este tipo de ecosistemas a nivel mundial (Keita 1987, Ratter et al. 1997, Bowman 1998, Cartron et al. 2005). La reducción de la cobertura arbustiva aumenta el lavado de nutrientes, disminuye la disponibilidad de agua, aumenta la escorrentía, reduce el establecimiento y crecimiento de plántulas, acelerando los procesos de erosión y desertificación (Shachak et al. 1998, Gutiérrez \& Squeo 2004). Las zonas áridas y semiáridas del norte-centro de Chile no están exentas de esta problemática (Prado et al. 1989, Campos-Ortega \& Jorquera-Jaramillo 2008, Squeo et al. 2001, 2008). El recurso arbóreo y arbustivo en el norte-centro de Chile fue intensamente explotado a partir del año 1600 para satisfacer la demanda creciente de energía de las incipientes actividades mineras (Folchi 2001, Santander 2003, Campos-Ortega \& JorqueraJaramillo 2008). Para cuantificar el proceso de deforestación ocurrido en el período 1600-1900 en el norte-centro de Chile, Santander (2003), sobre la base de una investigación con fuentes históricas, estima la producción de cobre metálico procesado con combustibles leñosos en cerca de $800 \mathrm{mil}$ toneladas para el período, el consumo total de leña seca requerida para esta producción la estima en 30 millones de toneladas, las cuáles fueron taladas en un $84,3 \%$ entre 1835 y 1900 (65 años), deforestando entre 3,36 y 6,73 millones de hectáreas.

Actualmente en la Región de Atacama, particularmente en la provincia de Huasco, se ha detectado un proceso sistemático de extracción de arbustos para la producción de carbón, el cual es comercializado en centros urbanos y localidades rurales. No obstante, no hay estimaciones sobre el total de carbón producido y el impacto que esto podría causar al ecosistema. Tampoco se ha investigado sobre las principales especies utilizadas. Observaciones en terreno permiten postular que los arbustos Adesmia hystrix Phil. (Fabaceae), Balsamocarpon brevifolium Clos. (Fabaceae) y Fabiana imbricata Ruiz et Pav. (Solanaceae) son los principales arbustos explotados.

El objetivo de la presente investigación fue estimar la producción de carbón a partir de arbustos nativos, así como identificar las principales especies utilizadas en este proceso en la provincia de Huasco, Región de Atacama.

\section{MATERIALES Y MÉTODO}

\section{Sitio DE ESTUdio}

La Región de Atacama $\left(26^{\circ} \mathrm{S}-29^{\circ} \mathrm{S}\right)$ se extiende entre la zona híperárida de la Región de Antofagasta y la zona mediterránea semiárida de la Región de Coquimbo. La región es dominada por un paisaje eco-geográfico andino (28,1\% de su superficie), serrano o cordillera de la Costa (21,3\%) y altiplánico (18,5\%), también destacan los paisajes denominados como preandino $(17,6 \%)$, pampeano $(9,6 \%)$, costero $(3,5 \%)$ y una zona de valles transversales $(1,4 \%)$ (Novoa et al. 2008). Presenta escasas precipitaciones, las que se concentran principalmente en los meses de invierno y aumentan con la altitud (Juliá et al. 2008). La región está bajo la influencia del ciclo oceanográfico-climático El Niño - Oscilación del Sur (ENOS) (Holmgren et al. 2006). En la zona norte-centro de Chile, la fase El Niño de esta oscilación se asocia a un significativo incremento en las precipitaciones (Montecinos \& Aceituno 2003). En el sur del desierto de Atacama, el incremento en la disponibilidad de agua es el factor que explica el aumento de la productividad primaria bajo los 2.500 m.s.n.m (Squeo et al. 2006b).

La flora vascular de la Región de Atacama comprende 980 especies nativas (Squeo et al. 2008) y posee uno de los mayores niveles de endemismos en el país: el 54,3\% de las especies nativas lo son para Chile y el 37,3\% para Atacama $\mathrm{y}$ regiones vecinas (regiones de Antofagasta y Coquimbo) (Letelier et al. 2008). La región se ubica en la porción norte del hotspot mundial de biodiversidad de Chile central llamado Chilean winter rainfall Valdivian forests, el cual 
alberga 3.893 especies de plantas nativas, con más de un $50 \%$ de endemismos al hotspot (Myers et al. 2000, Arroyo et al. 2004, Mittermeier et al. 2004).

LOCALIZACIÓN DE PUNTOS DE VENTA Y PRODUCCIÓN DE CARBÓN NATIVO

A partir de observaciones en terreno y trabajo de campo, se constató la presencia de centros de comercialización y producción de carbón principalmente en la provincia de Huasco, sur de la Región de Atacama, provincia que está conformada por cuatro comunas: Huasco, Vallenar, Alto del Carmen y Freirina (Fig. 1).

Durante los meses de enero y abril del año 2008 se detectaron los sitios de venta de carbón nativo en los principales centros poblados (ciudades y localidades rurales) del área de estudio. Posteriormente se estableció contacto con productores de carbón. Se realizaron 28 entrevistas estructuradas a vendedores, productores de carbón e informantes claves en 17 centros poblados (Fig. 1, Tabla I). Se detectaron 21 puntos de venta de carbón nativo, en cada uno se recolectaron muestras de entre 2 y 40 kilos de carbón.

\section{IDENTIFICACIÓN DE LAS ESPECIES UTILIZADAS}

La persistencia de un plano leñoso particular por especie es una característica de las formas de vida arbóreas (Montenegro 1985). Así mismo, los restos carbonizados conservan los criterios anatómicos de las maderas de origen, esto permite realizar procedimientos de identificación a partir de las muestras de madera carbonizada (Solari \& Lehnebach 2004).

Para la identificación botánica del carbón se elaboró un muestrario de referencia. Se recolectaron muestras leñosas de arbustos vivos, de las especies Balsamocarpon brevifolium ("algarrobilla"), Adesmia hystrix ("varilla brava") y Fabiana imbricata ("pichi", "romero"). Se cortaron secciones transversales de los tallos principales y secundarios, los que fueron posteriormente pulidos y observados bajo lupa, así se describió la estructura anatómica de esta sección de la planta en verde. Posteriormente, se realizó un proceso de carbonización de las muestras de referencia, siguiendo el protocolo de Solari \& Lehnebach (2004). Las muestras de referencias de los arbustos recolectados fueron secadas a temperatura ambiente. Luego, secciones de los tallos primarios y secundarios fueron envueltas en papel de aluminio (Alusa) y expuestas en un horno a $400^{\circ}$ durante 25 minutos. Finalmente, para determinar las especies utilizadas en la producción de carbón se compararon bajo lupa las muestras colectadas en los puntos de venta con las muestras de referencia.

FACTORES DE CONVERSIÓN (PESO LEÑA SECA - PESO CARBÓN) Y RENDIMIENTO POR SUPERFICIE (PESO LEÑA SECA / HECTÁREA) Para cuantificar el número de hectáreas con arbustos necesarios para la producción del carbón estimado, se requiere establecer previamente una relación de biomasa entre peso carbón y peso leña seca. Para la zona árida del sur del Sahara se estima que el rendimiento medio de la carbonización varía entre $16 \%$ y $30 \%$ del peso de la materia prima (i.e. cuociente peso carbón/peso leña seca entre 0,30 y 0,16) (Keita 1987). Otros autores señalan que para fabricar una tonelada de carbón vegetal se necesitan $6 \mathrm{~m}^{3}$ de leña seca (Wardle \& Palmieri 1981). El peso de un $\mathrm{m}^{3}$ de leña varía considerablemente, entre $200 \mathrm{~kg}$ en el caso de ramas retorcidas de arbustos sahelianos hasta $600 \mathrm{~kg}$ en el caso de troncos bien conformados obtenidos en operaciones de desmonte (Keita 1987); según estos datos, el cuociente peso carbón /peso leña seca varía entre 0,27 y 0,83 (Wardle \& Palmieri 1981). La FAO estima que para producir una tonelada de carbón vegetal se requiere entre 7 a $11 \mathrm{~m}^{3}$ de leña seca, considerando $535 \mathrm{~kg}$ por cada $\mathrm{m}^{3}$ de leña seca, el cuociente peso carbón / peso leña seca sería entre 0,17 y 0,27 (FAO 1983). Un estudio con Acacia caven (Molina) Molina, establece un rendimiento de 0,23 para la producción de carbón a base de leña seca (Pacheco 2005). Para este trabajo se considera un cuociente peso carbón /peso leña seca de 0,22 , el cual se encuentra dentro del rango de todos los valores reportados.

A partir del total de producción de carbón en el área de estudio es posible estimar el número de hectáreas deforestadas para estos requerimientos. Se debe establecer un rendimiento de toneladas de leña seca por hectárea (ton/ ha) para el área de estudio, lo cual va a estar asociado a la cobertura promedio de las especies analizadas y el peso promedio por planta. En una investigación con especies de Atriplex, se calcularon los siguientes rendimientos para las especies nativas de la Región de Atacama: A. atacamensis Phil. (3,01 ton/ha), A. clivicola I.M.Johnst. (0,41 ton/ha), A. deserticola Phil. (1,59 ton/ha), A. mucronata Phil. (0,14 ton/ha), con un rendimiento promedio para las cuatro especies de 1,28 ton/ha (Lailhacar et al. 1995). Prado et al. (1989) estiman una producción promedio de biomasa de 2,23 ton/ha, en catorce formaciones vegetales de la zona de Guatulame (comuna de Monte Patria, Región de Coquimbo). Considerando las condiciones más áridas del sector pampeano del área de estudio, se utilizó como valor inferior un rendimiento de 1,15 ton/ha (Tabla IV).

\section{RESULTADOS}

Se realizaron 28 entrevistas en las 4 comunas de la provincia de Huasco, cubriendo la totalidad del área de estudio (25\% de la Región de Atacama) (Tabla I). Las entrevistas fueron realizadas en 17 centros poblados (ciudades y localidades rurales) (Fig. 1). En 7 centros poblados $(41,2 \%)$ se localizaron un total de 21 puntos de ventas de carbón nativo, concentrándose en las ciudades: Vallenar (7), Huasco 
(4) Freirina (3), Alto del Carmen (2), Cachiyuyo (2), El Tránsito (2), Domeyko (1). Del total de 28 entrevistados, 12 son comerciantes $(42,9 \%), 6$ crianceros caprinos $(21,4 \%)$, 2 mineros $(7,1 \%), 2$ agricultores $(7,1 \%)$ y 2 presidentes de junta de vecinos $(7,1 \%)$ y 4 de otras actividades $(14,3 \%)$. De éstos, 14 vendían carbón nativo al momento de la entrevista (50\%), los 12 comerciantes y dos crianceros. En tres casos, los vendedores eran a su vez productores de carbón y fueron localizados a través de otros vendedores o informantes claves. De los 14 vendedores, 4 de ellos $(28,6 \%)$ conocían en detalle sobre las prácticas de producción de carbón, los 10 vendedores restantes sólo pudieron dar información respecto a sus propias ventas.

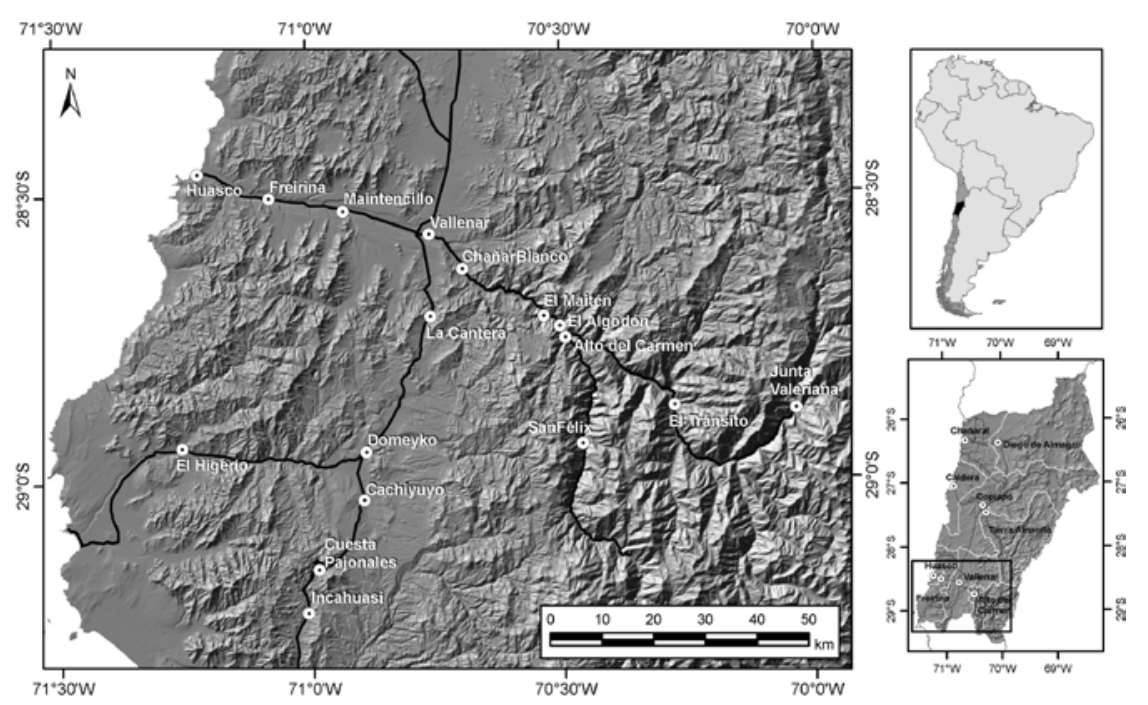

FigURA 1. Área de estudio y centros poblados donde se realizaron las entrevistas y recolectaron las muestras de carbón nativo comercializadas. En el mapa de ubicación de la Región de Atacama se muestran las capitales comunales (puntos), límites comunales (líneas blancas) y red vial básica (líneas negras).

FIGURE 1. Studied area and populated centers where the interviews were made and commercial native charcoal samples were collected. In the location map of the Atacama Region (main cities (points), county borders (white lines) and main routes (black lines) are showed.

TABLA I. Número y actividad de entrevistados, y presencia de puntos de venta de carbón nativo en cada localidad estudiada.

TABLE I. Number and activity of the interviewers and, presence of sales points of native charcoal for each locality studied.

\begin{tabular}{clcccl}
\hline \multirow{2}{*}{$\mathrm{N}^{\circ}$} & Ciudad o localidad & Comuna & Venta carbón & & \\
\cline { 4 - 6 } & & nativo & $\mathrm{n}$ & & \multicolumn{1}{c}{ Entrevistados } \\
\hline 1 & Incahuasi & Vallenar & no & 3 & Minería, comercio, criancería caprina. \\
2 & Pajonales & Vallenar & no & 1 & Agricultura. \\
3 & Cachiyuyo & Vallenar & si & 2 & Minería, presidente junta de vecinos. \\
4 & Domeyko & Vallenar & si & 2 & Mecánica, comercio. \\
6 & La Cantera & Vallenar & no & 2 & Presidente junta de vecinos, criancería caprina. \\
7 & Vallenar & Vallenar & si & 4 & Comercio, criancería caprina, producción de carbón. \\
8 & Maitencillo & Freirina & no & 1 & Comercio. \\
5 & El Higirío & Freirina & no & 1 & Criancería caprina. \\
9 & Freirina & Freirina & si & 2 & Comercio. \\
10 & Huasco & Hi & 2 & Comercio. \\
11 & Chañar Blanco & Alto del Carmen & no & 1 & Agricultura. \\
12 & El Maitén & Alto del Carmen & no & 1 & Criancería caprina. \\
13 & El Algodón & Alto del Carmen & no & 1 & Agricultura. \\
14 & Alto del Carmen & Alto del Carmen & si & 1 & Comercio. \\
15 & El Tránsito & Alto del Carmen & si & 1 & Comercio. \\
16 & Junta de Valeriano & Alto del Carmen & no & 2 & Criancería caprina, presidente de junta de vecinos. \\
17 & San Félix & Alto del Carmen & no & 1 & Comercio. \\
\hline
\end{tabular}


ESPECIES UTILIZADAS PARA LA PRODUCCIÓN DE CARBÓN La identificación de las especies utilizadas para la producción de carbón se realizó a partir de las observaciones y descripciones de las muestras de referencia (Fig. 2). En Fabiana imbricata se observa gran cantidad de glándulas resinosas aromáticas en todos los tejidos del tallo, las cuales se mantienen luego del proceso de carbonización. Los anillos de crecimiento son claramente identificables. Los radios del xilema son delgados y rectos entre los anillos de crecimiento. En Balsamocarpon brevifolium no se observan glándulas resinosas. Los anillos de crecimiento son más difusos con respecto a la especie anterior. Los radios son rectos y relativamente más gruesos, con espacios interradiales angostos y regulares. En Adesmia hystrix tampoco se observan glándulas resinosas. Los anillos de crecimiento son más difusos que las especies anteriores. Los radios son gruesos, ondulados y con espacios interradiales amplios e irregulares.

Fabiana imbricata es la especie más utilizada para la producción de carbón, la cual está presente en 13 de las 21 muestras (61,9\%) (Tabla II). Balsamocarpon brevifolium fue encontrada en 5 muestras $(23,8 \%)$ y se detectó Adesmia hystrix en 4 muestras (19,0\%). En 2 muestras (9,5\%) no fue posible identificar ninguna de las tres especies estudiadas, lo que sugiere que hay otras especies utilizadas en la fabricación de carbón.

Producción AnUal de CARBón NATIVo EN LA zona de ESTUdio Se detectaron 4 grupos productivos en la provincia de Huasco, en los cuales trabajan un total aproximado de 42 personas, desde la extracción de la leña al traslado del carbón a los centros de comercialización (Tabla III). Dos grupos corresponden a pequeñas unidades productivas de 3 personas, asociadas a unidades familiares; otro grupo está conformado por 9 personas (3 familias asociadas); el cuarto grupo lo integran 27 personas, constituyendo una pequeña-empresa informal. Según la información recopilada, la producción total de estos 4 grupos fue de 3.510 sacos de carbón nativo para el 2007, lo que equivale aproximadamente a 134.258 $\mathrm{kg}$ de carbón. Se detectó una variación entre grupos respecto a la producción total de carbón, lo que se traduce en una producción per cápita anual que varía entre 2.467 a 4.200 kg por persona. Aunque se presenta la producción en base anual, los entrevistados señalaron que la temporada de producción varía entre 3 y 6 meses.

EXTRACCIÓN ANUAL DE ARBUSTOS PARA LA PRODUCCIÓN DE CARBÓN NATIVO

Las cuatro unidades productivas utilizaron aproximadamente $610.263 \mathrm{~kg}$ de leña seca para la producción de $134.258 \mathrm{~kg}$ de carbón en el año 2007 (i.e., cuociente peso carbón /peso leña seca igual a 0,22 ). Con un rendimiento de 1,15 ton/ha se calcula una deforestación aproximada de 530,7 ha con arbustos. El CENSO 2002 registra 88 personas en la provincia de Huasco que señalan dedicarse a la silvicultura o extracción de madera como principal actividad. A un rendimiento de 1,15 ton/ha, se habrían deforestado 1.112 hectáreas con arbustos el año 2007. Considerando que el CENSO 2002 señala que la Región de Atacama posee un total de 209 personas dedicadas a la extracción de carbón, se estima que esta región produciría $668.093 \mathrm{~kg}$ de carbón vegetal. Lo anterior requeriría 3.036.788 $\mathrm{kg}$ de leña seca y se explotaría una superficie arbustiva de entre 2.641 a 1.362 ha por año (considerando un rendimiento de 1,15 a 2,23 ton/ha) (Tabla IV).

INGRESO BRUTO TOTAL Y PER CÁPITA

El ingreso bruto estimado para cada uno de los cuatro grupos productivos se basó en el precio de mercado mayorista de $\$ 10.000$ por saco (valor del primer semestre del 2008 en la Provincia de Huasco), independiente del peso exacto y el tipo de carbón (ver Tabla V). Este es el valor por el cual el productor vende el saco a un intermediario. Los 3.510 sacos o $134.258 \mathrm{~kg}$ de carbón nativo generan un ingreso total de $\$ 35.100 .000$ a los productores, es decir a un promedio de $\$ 261$ el kg de carbón nativo. Según estos cálculos, el promedio de ingreso bruto (sin descontar los costos de producción) anual por persona varía entre $\$ 622.222$ y $\$ 1.000 .000$, con un promedio de $\$ 835.714$. Recalculados estos valores en una base mensual per cápita durante los meses trabajados en la actividad, el ingreso bruto mensual per cápita es entre los \$222.222 y los \$124.444 mensuales, con un promedio de $\$ 174.475$. Estos valores se encuentran cercanos al ingreso mínimo oficial durante el mismo periodo (i.e., \$135.000 hasta junio de 2008). El precio final de venta a público en los centros urbanos o localidades rurales de un $\mathrm{kg}$ de carbón se encontraba entre $\$ 600$ y $\$ 800$ durante el primer semestre de 2008. Esto resulta en una relación precio mercado / precio productor de 2,29 a 3,06.

\section{UNIDADES PRODUCTIVAS}

A partir de los datos recogidos en terreno es posible distinguir entre 3 tipos de formas asociativas en la producción de carbón vegetal. En primer lugar, una unidad productiva organizada en torno a la familia, asociada mayormente al mundo agropecuario, los cuales producen un bajo nivel de impacto al ecosistema (entre 8 y 29 ha/año). Este grupo se vincula con la actividad basada en una economía de subsistencia, con un ingreso bruto cercano a los $\$ 2.000 .000$ por familia por temporada. En segundo lugar, una asociación de familias que se organizan con el objetivo de producir carbón vegetal de un modo más eficiente compartiendo recursos como mano de obra, animales y vehículos. El nivel de impacto al ecosistema de este grupo es regular (entre 30 y 100 ha/año) y también estaría asociado a una economía de subsistencia con un ingreso bruto cercano a los $\$ 6.000 .000$ en conjunto. Por último, una forma asociativa tipo microempresa con trabajadores asalariados (más de 25 personas), más vinculados a los centros urbanos que al mundo agropecuario, con un ingreso bruto cercano a los $\$ 25.000 .000$ en conjunto por temporada. Este grupo presenta un nivel de impacto alto (entre 101 y 324 ha/año). 

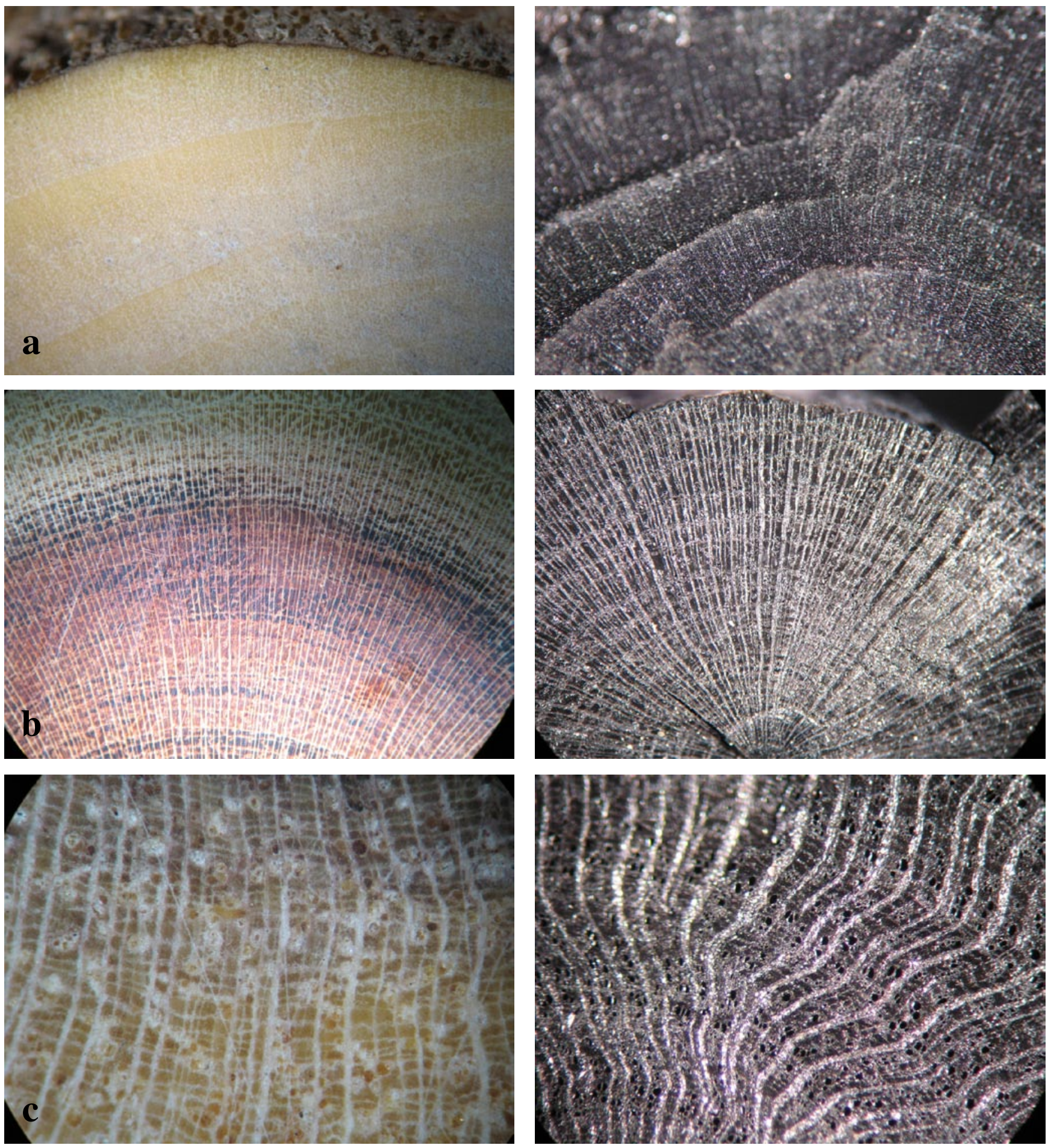

FiguRA 2. Muestras de cortes transversales de tallos de arbustos verdes (derecha) y carbonizados (izquierda): a. Fabiana imbricata Ruiz et Pav. (Solanaceae); b. Balsamocarpon brevifolium Clos. (Fabaceae); c. Adesmia hystrix Phil. (Fabaceae).

FigURE 2. Samples of cross sections of shrubs from green (right) and carbonized (left) stems: a. Fabiana imbricata Ruiz et Pav. (Solanaceae); b. Balsamocarpon brevifolium Clos. (Fabaceae); c. Adesmia hystrix Phil. (Fabaceae). 
Producción de carbón vegetal en la Región de Atacama: Estevez, R. ET AL.

TABLA II. Especies de arbustos nativos utilizadas en la producción de carbón según muestras recolectadas en los puntos de venta.

TABLE II. Native shrub species used on the charcoal production based on collected samples in each sales point.

\begin{tabular}{lcc}
\hline \multirow{2}{*}{ Especies } & \multicolumn{2}{c}{ Muestras } \\
\cline { 2 - 3 } & $\mathrm{n}$ & $\%$ \\
\hline F. imbricata & 10 & 47,6 \\
B. brevifolium & 3 & 14,3 \\
A. hystrix & 3 & 14,3 \\
F. imbricata y B. brevifolium & 2 & 9,5 \\
F. imbricata y A. hystrix & 1 & 4,8 \\
Otros no determinados & 2 & 9,5 \\
\hline Total & 21 & 100,0 \\
\hline
\end{tabular}

TABLA III. Producción total de carbón por grupos productivos en la provincia de Huasco. Se indica el número de personas que trabajan en la producción de carbón, el peso de un saco de carbón, la cantidad estimada de sacos producidos por año, la producción total y la producción per cápita.

TABLE III. Total charcoal production by productivity groups on the Huasco province. Number of people that work on the charcoal production, charcoal bag weight, estimated number of bag produced by year, total production and, per capita production are showed.

\begin{tabular}{lcccccc}
\hline Grupos productivos & $\begin{array}{c}\text { Personas que } \\
\text { trabajan }\end{array}$ & $\begin{array}{c}\text { Saco } \\
(\mathrm{kg})\end{array}$ & $\begin{array}{c}\text { Producción Saco } \\
/ \text { año }\end{array}$ & $\begin{array}{c}\text { Producción Total } \\
(\mathrm{kg})\end{array}$ & $\begin{array}{c}\text { Saco-año / } \\
\text { persona }\end{array}$ & $\begin{array}{c}\text { Producción per } \\
\text { cápita } \\
(\mathrm{kg} / \mathrm{persona})\end{array}$ \\
\hline Vallenar 1* & 3 & 37 & 200 & 7.400 & 66,7 & 2.467 \\
Incahuasi & 3 & 42 & 300 & 12.600 & 100,0 & 4.200 \\
El Tránsito & 9 & 37 & 560 & 20.720 & 62,2 & 2.302 \\
Vallenar 2* & 27 & 37 & 2.450 & 90.650 & 90,7 & 3.357 \\
Total & 42 & 38,25 & 3.510 & 134.258 & 83,6 & 3.197 \\
\hline
\end{tabular}

* En la ciudad de Vallenar fueron contactados dos productores con distintas características / In Vallenar city we contacted two producers with different characteristics

TABLA IV. Hectáreas deforestadas según rendimiento (ton/ha) y número total de productores.

TABLE IV. Deforested hectares by yield (tons/ha) and total number of producers.

\begin{tabular}{|c|c|c|c|c|c|}
\hline \multirow{2}{*}{$\begin{array}{l}\text { Número de productores } \\
\quad \text { (personas) }\end{array}$} & \multicolumn{2}{|c|}{ Producción (kg) } & \multicolumn{3}{|c|}{$\begin{array}{l}\text { Hectáreas deforestadas según rendimientos } \\
\text { (ton/ha) }\end{array}$} \\
\hline & Carbón & Leña seca & 1,150 & 2,230 & 3,345 \\
\hline 1 & $3.196,6$ & $14.530,0$ & 12,6 & 6,5 & 4,3 \\
\hline $42^{\mathrm{a}}$ & 134.258 & $610.263,6$ & 530,7 & 273,7 & 182,4 \\
\hline $88^{\mathrm{b}}$ & $281.302,5$ & $1.278 .647,7$ & $1.111,9$ & 573,4 & 382,3 \\
\hline $209^{c}$ & $668.093,4$ & $3.036 .788,2$ & $2.640,7$ & $1.361,8$ & 907,9 \\
\hline $300^{\mathrm{d}}$ & $958.985,7$ & $4.358 .903,1$ & $3.790,3$ & $1.954,7$ & $1.303,1$ \\
\hline
\end{tabular}

${ }^{a}$ En los cuatro centros de producción investigados / In the four production center studied

${ }^{\mathrm{b}}$ En la provincia de Huasco (CENSO 2002) / In the Huasco Province

${ }^{\mathrm{c}}$ En la Región de Atacama (CENSO 2002) / In the Atacama Region

d Proyectado en base a información disponible en la Región de Atacama / Projected in base to available information for the Atacama Region 
TABLA V. Ingreso bruto anual y por persona según grupo de productor. El valor de mercado de un saco de carbón fue de \$10.000 (pesos chilenos) a nivel de productor (primer semestre del 2008).

TABLE V. Annual gross income and per person by productivity groups. The market value of one charcoal bag was $\$ 10.000$ (Chilean pesos) in the producer level (first half of 2008).

\begin{tabular}{cccccccc}
\hline $\begin{array}{c}\text { Grupos } \\
\text { productivos }\end{array}$ & $\begin{array}{c}\text { Personas } \\
\text { que } \\
\text { trabajan }\end{array}$ & $\begin{array}{c}\text { Producción Saco } \\
\text { / año }\end{array}$ & $\begin{array}{c}\text { Total ingreso bruto } \\
\text { anual }\end{array}$ & $\begin{array}{c}\text { Ingreso bruto } \\
\text { anual / persona }\end{array}$ & $\begin{array}{c}\text { Valor } \\
1 \mathrm{~kg} \\
\text { carbón }\end{array}$ & $\begin{array}{c}\text { Meses de } \\
\text { trabajo }\end{array}$ & $\begin{array}{c}\text { Ingreso } \\
\text { mensual per } \\
\text { cápita* }\end{array}$ \\
\hline Vallenar 1** & 3 & 200 & $\$ 2.000 .000$ & $\$ 666.667$ & $\$ 270$ & 3 & $\$ 222.222$ \\
Incahuasi & 3 & 300 & $\$ 3.000 .000$ & $\$ 1.000 .000$ & $\$ 238$ & 5 & $\$ 200.000$ \\
El Tránsito & 9 & 560 & $\$ 5.600 .000$ & $\$ 622.222$ & $\$ 270$ & 5 & $\$ 124.444$ \\
Vallenar 2** & 27 & 2450 & $\$ 24.500 .000$ & $\$ 907.407$ & $\$ 270$ & 6 & $\$ 151.235$ \\
\hline Total & 42 & 3510 & $\$ 35.100 .000$ & $\$ 835.714$ & $\$ 261$ & & $\$ 174.475$ \\
\hline
\end{tabular}

* Ingreso mensual per cápita durante los meses de trabajo en la actividad / per capita monthly income during the working months in the activity

** En la ciudad de Vallenar fueron contactados dos productores con distintas características / In Vallenar city we contacted two producers with different characteristics

\section{DISCUSIÓN Y CONCLUSIONES}

Enestetrabajohasidoposibleconstatarunprocesosistemático y actual de producción de carbón vegetal a partir de arbustos nativos en la provincia de Huasco, Región de Atacama. La fabricación de carbón es principalmente a base de Fabiana imbricata, Balsamocarpon brevifolium y Adesmia hystrix. Las tres especies pueden alcanzar estados de adultez por sobre los $2 \mathrm{~m}$ de altura con tallos lignificados y maderas duras aptas para la producción de carbón. Este trabajo presenta desafíos para establecer el estado de conservación de las especies explotadas, sólo Balsamocarpon brevifolium está actualmente catalogada como vulnerable, en tanto Fabiana imbricata y Adesmia hystrix están consideradas como sin problemas de conservación (Squeo et al. 2008). La constatación de procesos sistemáticos de extracción son antecedentes que pueden considerarse para reevaluar el actual estado de conservación de las especies estudiadas.

Se detectó la existencia de al menos 4 centros de producción con un total de $134.258 \mathrm{~kg}$ de carbón producidos en el año 2007, en un proceso que participaron 42 personas. El total de hectáreas de arbustos explotadas sería entre 531 y 274 ha por año. El catastro de uso del suelo y vegetación de CONAF (1999) indica que la provincia de Huasco posee 874.050 ha de Matorral y 556.006 ha de Matorral con Suculentas. Considerando este universo (1.420.000 há), la superficie equivalente explotada por los cuatro grupos de productores estudiados va entre un $0,02 \%$ y un $0,04 \%$ anual. Considerando una estimación de 88 personas dedicadas a la producción de carbón en la provincia de Huasco (CENSO 2002), se explotarían anualmente entre el $0,04 \%$ y $0,08 \%$ de la superficie de Matorral y Matorral con Suculentas. Los datos de entrevistas sugieren que el número de productores de carbón en la provincia de Huasco es más del doble a la entregada por el CENSO 2002. Con 200 productores, la superficie equivalente explotada podría llegar anualmente hasta 2.527 ha $(0,18 \%$ de la superficie con Matorrales y Matorrales con Suculentas), bajo este escenario en 100 años sería consumido totalmente el recurso arbustivo en la provincia de Huasco. Esta tasa de deforestación es menor a la estimada por Santander (2003) para el período 1600-1900 en el norte-centro de Chile. La recuperación de las poblaciones de arbustos explotadas es incierta, particularmente para Balsamocarpon brevifolium.

La recuperación de la cubierta vegetal en esta región, cuando ésta ocurra, sería con especies de mayor velocidad de crecimiento y menos apetecidas para la producción de carbón, por lo que se esperaría una selección negativa en contra de las especies explotadas. Las implicancias ecológicas de esta selección negativa conllevarían un cambio en la composición y estructura de las comunidades biológicas afectadas, y probablemente una disminución progresiva de la cubierta vegetal arbustiva. Adicionalmente, otros factores de perturbación estarían operando en estas comunidades naturales (e.g., ingreso de especies invasoras, sobre pastoreo, fragmentación del hábitat), lo que reduce la posibilidad de su recuperación natural (Squeo et al. 2008, 2009).

Las estimaciones realizadas pueden tener variaciones asociadas a factores climáticos y económicos. En años secos, el impacto de la escasez de agua sobre la productividad vegetal afecta directamente a las poblaciones rurales del norte-centro de Chile dedicadas a actividades agropecuarias, lo que genera un aumento de las personas dedicadas a la producción de carbón. Así mismo, los períodos de baja 
del precio internacional del cobre generan inestabilidad laboral en la población dedicada a actividades mineras, lo que también conlleva un aumento en la producción de carbón vegetal. En este sentido, un problema ecológico, como la explotación de arbustos en zonas áridas, se vincula a una situación de vulnerabilidad social frente a variaciones climáticas y económicas que afectan a poblaciones en situación de riesgo social.

La protección del recurso arbustivo es un aspecto crítico para la conservación de la biodiversidad en el norte-centro de Chile. A partir de los resultados presentados, las estrategias de manejo debieran considerar la producción de carbón como una de las principales amenazas para estas especies. No obstante, es necesario diferenciar las unidades productivas y establecer control especialmente en aquellos casos donde el nivel de impacto es alto (por ejemplo, más de $150 \mathrm{ha} / \mathrm{año}$ ). Pequeños productores, con niveles de impactos bajos y relacionados al mundo agropecuario, que establecen una relación de subsistencia con su entorno natural, no debieran ser el foco de atención de las políticas al respecto. Por otra parte, las acciones concretas deben considerar no sólo la regulación de la extracción, sino también controlar la demanda del carbón nativo local en los centros urbanos. Se observó que los principales consumidores del carbón vegetal a partir de arbustos nativos viven en centros urbanos y éste es utilizado en su mayoría para consumo doméstico. En el mercado es posible encontrar carbón vegetal del centro-sur de Chile; sin embargo, el menor poder calórico de éste, en comparación con las maderas duras de los arbustos nativos estudiados, lo hace menos demandado. A partir de los datos obtenidos, se calcula en la Región de Atacama un consumo de 2,7 kg de carbón nativo por persona al año (considerando una población de 244.336 personas). Es necesario entonces informar y educar a la población para que opte por el carbón del centro-sur de Chile, a pesar de su menor calidad y así reducir la demanda y producción de carbón a partir de arbustos nativos locales.

\section{AGRADECIMIENTOS}

Este trabajo es parte de la tesis de Magíster en Ciencias Biológicas mención Ecología de Zonas Áridas (Universidad de La Serena) del primer autor, quien agradece a la Beca de Magíster IEB-2007 (ICM P05-002) y a la Beca de Magíster CONICYT (2008). Este estudio fue financiado por proyectos FNDR- Región de Atacama (Código BIP: 30057872-0), P05-002 ICM de MIDEPLAN y CONICYT PFB-23.

\section{BIBLIOGRAFÍA}

Aguiar, M.R. \& O.E. Sala. 1999. Patch structure, dynamics and implications for the functioning of arid ecosystems. Trends in Ecology and Evolution 14(7): 272-277.

Armesto, J.J., P.E. Vidiella \& J.R. Gutiérrez. 1993. Plant communities of the fog-free coastal desert of Chile: plant strategies in a fluctuating environment. Revista Chilena de Historia Natural 66: 271-282.

Arroyo, M.T.K., P. Marquet, C. Marticorena, J. Simonetti, L. Cavieres, F.A. Squeo \& R. Rozzi. 2004. Chilean winter rainfall-valdivian forests. In: A. Mittermeier, P. Robles, M. Hoffmann, J. Pilgrin, T. Brooks, C.G. Mittermeier, J. Lamoreux \& G.A.B. da Fonseca (eds.), Hotspots revisited, pp. 99-103. CEMEX, México D.F., México.

Bowman, D.M.J.S. 1998. Tansley review No. 101. The impact of aboriginal landscape burning on the Australian biota New Phytologist 140(3): 385-410.

Caldwell, M.M., T.E. Dawson \& J.H. Richards. 1998. Hydraulic lift: consequences of water efflux from the roots of plants. Oecologia 113(2): 151-161.

Campos-Ortega, C. \& C. Jorquera-Jaramillo. 2008. Minería y conservación en Atacama. En: F.A. Squeo, G. Arancio \& J.R. Gutiérrez (eds.), Libro rojo de la flora nativa y de los sitios prioritarios para su conservación: Región de Atacama, pp. 323-338. Ediciones Universidad de La Serena, La Serena, Chile.

Cartron, J.L.R., G. Ceballos \& R.S. Felger. 2005. Biodiversity, Ecosystems, and Conservation in Northern Mexico. Oxford University Press, New York. 496 pp.

CONAF. 1999. Catastro y Evaluación de los Recursos Vegetacionales Nativos de Chile. Informe Nacional con Variables Ambientales. Comisión Nacional Forestal, Santiago. 88 pp.

FAO. 1983. Métodos simples para fabricar carbón vegetal. Organización de las Naciones Unidas para la Agricultura y la Alimentación, Roma. 157 pp.

FolchI, M. 2001. La insustentabilidad de la industria del cobre en Chile: los hornos y los bosques durante el siglo XIX. Revista Mapocho (Chile) 49: 149-175.

Fuentes, E.R. 1988. Sinopsis de paisajes de Chile central. En: E.R. Fuentes \& S. Prenafeta (eds.), Ecología del paisaje en Chile Central, pp. 17-28. Ediciones Universidad Católica de Chile, Santiago, Chile.

Fuentes, E.R., R.D. Otaiza, M.C. Alliende, A. Hoffmann \& A. PoIANI. 1984. Shrub clumps of the chilean matorral vegetation: structure and possible maintenance mechanisms. Oecologia 62(3): 405-411.

GutiérReZ, J.R. \& F.A. SQueO. 2004. Importancia de los arbustos en los ecosistemas semiáridos de Chile. Ecosistemas 13(3): 36-45.

Gutiérrez, J.R., P.L. Meserve, J.M. Jaksic, L.C. Contreras, S. Herrera \& H. VÁsquez. 1993. Structure and dynamics of vegetation in a Chilean semiarid thornscrub community. Acta Oecologia 14: 271-285.

Holmgren, M., P. Stapp, C.R. Dickman, C. Gracia, S. Graham, J.R. Gutiérrez, C. Hice, F. Jaksic, D.A. Kelt, M. Letnic, M. Lima, B.C. López, P.L. Meserve, W.B. Milstead, G.A. Polis, M.A. Previtali, M. Richter, S. Sabat \& F.A. SQUEO. 2006. A synthesis of ENSO effects on drylands in Australia, North America and South America. Advances in Geosciences 6: 69-72.

Horton, J.L. \& S.C. HART. 1998. Hydraulic lift: a potentially important ecosystem process. Trends in Ecology and Evolution 13(6): 232-235.

Juliá, C., S. Montecinos \& A. Maldonado. 2008. Características 
climáticas de la Región de Atacama. En: F.A. Squeo, G. Arancio \& J.R. Gutiérrez (eds.), Libro rojo de la flora nativa y de los sitios prioritarios para su conservación: Región de Atacama, pp. 25-42. Ediciones Universidad de la Serena, La Serena, Chile.

KeITA, J.D. 1987. Leña o carbón vegetal: ¿Qué solución es la mejor? Unasylva (Italia) 39: 61-66.

Lailhacar, S., H. Rivera, H. Silva \& J. Caldentey. 1995. Rendimiento de leña y recuperación al corte en diferentes especies y procedencias arbustivas del género Atriplex. Revista de Ciencias Forestales (Chile) 10: 85-97.

LEÓN, M.F. \& F.A. SQuEO. 2004. Levantamiento hidráulico: la raíz del asunto. En: H.M. Cabrera (ed.), Fisiología Ecológica en Plantas, pp. 99-109. Ediciones Universidad de Valparaíso, Valparaíso, Chile.

Letelier, L., F.A. Squeo, G. Arancio, A. Marticorena, M. MuñozSchick, M.T.K. Arroyo, P. León-Lobos, S. Montecinos \& J.R. GutiérRez. 2008. Diversidad vegetal de la Región de Atacama, Chile. En: F.A. Squeo, G. Arancio \& J.R. Gutiérrez (eds.), Libro rojo de la flora nativa y de los sitios prioritarios para su conservación: Región de Atacama, pp. 123-135. Ediciones Universidad de La Serena, La Serena, Chile.

Mittermeier, R.A., P.R. Gil, M. Hoffmann, J. Pilgrim, T. Brooks, C.G. Mittermeier, J. Lamoreux \& G.A.B. DA Fonseca. 2004. Hotspots Revisted: Earth's Biologically Wealthiest and most Threatened Ecosystems. CEMEX, México D.F. 392 pp.

Montecinos, A. \& P. Aceituno. 2003. Seasonality of the ENSOrelated rainfall variability in central Chile and associated circulation anomalies. Journal of Climate 16(2): 281-296.

Montenegro, G. (ed.). 1985. Manual de técnicas de estudio estructural y ultraestructural en vegetales. Pontificia Universidad Católica de Chile, Santiago. 89 pp.

Myers, N., R.A. Mittermeier, C.G. Mittermeier, G.A.B. DA Fonseca \& J. Kent. 2000. Biodiversity hotspots for conservation priorities. Nature 403(6772): 853-858.

Novoa, J.E., Y. Tracol \& D. LóPez. 2008. Paisajes eco-geográficos de la región de Atacama. En: F.A. Squeo, G. Arancio \& J.R. Gutiérrez (eds.), Libro rojo de la flora nativa y de los sitios prioritarios para su conservación: Región de Atacama, pp. 13-24. Ediciones Universidad de La Serena, La Serena, Chile.

PACHECO, G. 2005. Evaluación del proceso de carbonización y calidad del carbón de Acacia caven (Mol.) Mol. producido en hornos de barro. Disertación, Universidad de Chile, Chile.

Prado, J.A., P. Infante, M. Arriagada \& S. Aguirre. 1989. Aprovechamiento de la biomasa en zonas áridas y semiáridas. CONAF-PNUD-FAO, Santiago. $61 \mathrm{pp}$.
Ratter, J.A., J.F. Ribeiro \& S. Bridgewater. 1997. The Brazilian Cerrado Vegetation and threats to its biodiversity. Annals of Botany 80: 223-230.

SANTANDER,A. 2003. Norte Chico (Chile). Consumo de combustibles en el procesamiento de los minerales de cobre y su impacto sobre los recursos arbóreos y arbustivos: 1601-1900. En: P. Livenais \& X. Aranda (eds.), Dinámicas de los sistemas agrarios en el Chile árido: la Región de Coquimbo, pp. 159-177. LOM Ediciones, Santiago, Chile.

ShachaK, M., M. Sachs \& I. Moshe. 1998. Ecosystem management of desertified shrublands in Israel. Ecosystems 1(5): 475483.

Solari, M.E. \& C. Lehnebach. 2004. Pensando la antracología para el centro-sur de Chile: sitios arqueológicos y bosque en el lago Calafquén. Chungara (volumen especial): 373380 .

Squeo, F.A., N. Olivares, S. Olivares, A. Pollastri, E. Aguirre, R. Aravena, C. Jorquera \& J.R. Ehleringer. 1999. Grupos funcionales en arbustos desérticos del norte de Chile, definidos sobre la base de las fuentes de agua utilizadas. Gayana Botánica 56(1): 1-15.

Squeo, F.A., G. Arancio \& J.R. Gutiérrez (eds.). 2001. Libro rojo de la flora nativa y de los sitios prioritarios para su conservación: Región de Coquimbo. Ediciones Universidad de La Serena, La Serena. 372 pp.

Squeo F.A., R. Aravena, E. Aguirre, A. Pollastri, C.B. Jorquera \& J.R. Ehleringer. 2006a. Groundwater dynamics in a coastal aquifer in North-central Chile: Implications for groundwater recharge in an arid ecosystem. Journal of Arid Environments 67: 240-254.

Squeo, F.A., Y. Tracol, D. López, J.R. Gutiérrez, A.M. Córdova \& J.R. Ehleringer. 2006b. ENSO effects on primary productivity in Southern Atacama Desert. Advances in Geosciences 6: 273-277.

Squeo, F.A., G. Arancio \& J.R. Gutiérrez (eds.). 2008. Libro rojo de la flora nativa y de los sitios prioritarios para su conservación: Región de Atacama. Universidad de La Serena, La Serena. 466 pp.

Squeo, F.A., Y. Tracol, D. López, M. León \& J.R. Gutiérrez. 2009. Vegetación nativa y variación temporal de la productividad en la provincia del Elqui. En: J. Cepeda (ed.), Los Sistemas Naturales de la Cuenca del Río Elqui (Región de Coquimbo, Chile): Vulnerabilidad y Cambio del Clima, pp. 162-185. Ediciones Universidad de La Serena, La Serena, Chile.

Wardle, P. \& M. Palmieri. 1981. ¿Cuál es el costo real de la leña? Unasylva (Italia) 33: 20-23.

Recibido: 13.10 .09

Aceptado: 27.05 .10 